\title{
Empowering the Caregiver Network of Farmers With a Disability: A Case Study of the North Carolina AgrAbility Project
}

\author{
K. Minus ${ }^{1}$, J. Parker Woods², R. Roberts ${ }^{3}$, C. Warren English ${ }^{4}$, B. M. Rodriguez ${ }^{5}$
}

\section{Abstract}

A majority of farmers with a disability rely heavily on caregivers to ensure they can fulfill their daily roles and responsibilities. Family members, such as spouses, parents, siblings, and children, are the most common caregivers. However, little is known about the resources and support needed to ensure these individuals can successfully navigate this complex role. In response, the North Carolina AgrAbility Project has provided caregivers with education, resources, and support so that these individuals can better assist farmers with a disability to minimize the job-related obstacles they face. In the current study, we sought to examine how caregivers of farmers with a disability have been empowered through the North Carolina AgrAbility Project. When viewed through the lens of Zimmerman's empowerment theory, four themes emerged (a) barriers to empowerment; (b) intrapersonal empowerment; (c) interactional empowerment; and (d) behavioral empowerment. Consequently, findings from this investigation documented that caregivers navigated key barriers to become empowered after receiving assistance from the North Carolina AgrAbility Project. Further, their experiences in AgrAbility changed how they approached supporting farmers with a disability. In response, we provide recommendations for better supporting and leveraging the caregiver network of farmers with a disability.

\section{Keywords}

accommodations, assistive technology, professional development, qualitative research, special needs

1. Kristoff T. Minus, Agriculture Teacher and FFA Advisor, Mac Williams Middle School, 4644 Clinton Rd, Fayetteville, NC, 28312, kristoffminus@ccs.k12.nc.us, (D https://orcid.org/0000-0001-5127-443X

2. S. Janine Parker Woods, Associate Administrator for Cooperative Extension, Virginia State University, 1 Hayden Drive, P.O. Box 9081, Virginia State University, VA 23806,jpwoods@vsu.edu, ID https://orcid.org/0000-0001-9296-5106

3. 3Richie Roberts, Assistant Professor, Louisiana State University, 131 J.C. Miller Baton Rouge, LA 70803, roberts3@lsu.edu, iD https://orcid.org/0000-0002-2993-4945

4. Chastity Warren English, Associate Professor, North Carolina Agricultural and Technical State University, 1601 N. Market Street, 251A Carver, Greensboro, NC, 27411 ckwarren@ncat.edu, Dhttps://orcid.org/0000-0003-4474-1495

5. Beatriz M. Rodriguez, Extension Associate - NC AgrAbility and NC Agromedicine, North Carolina Agricultural and Technical State University, 107 Coltrane Hall, P. O. Box 21928, Greensboro, NC 27420, bmrodrig@ncat.edu, https://orcid.org/0000-0002-0932-7523 


\section{Introduction and Problem Statement}

There are approximately 288,000 individuals in the U.S. involved in the agricultural industry between the ages of 15 and 79 who have a disability, which complicates their ability to fulfill their job-related duties (Fetsch et al., 2020b). Some of the major difficulties farmers with a disability face include: (a) the lack of information about adequate worksite accommodations; (b) economic constraints caused from wage loss and a lack of insurance; (c) residing in isolated areas that lack necessary services, and (d) limited professionals who have the knowledge needed to successfully accommodate their disability (Fetsch et al., 2020a; Fetsch \& Turk, 2018).

One program designed to assist farmers with a disability with job-related accommodations is the National AgrAbility Project (2021), founded in 1991 it provides education, resources, and support to individuals with disabilities to ensure they can succeed in their daily lives. The National AgrAbility Project was authorized under the Food, Agriculture, and Trade Act of 1990 and administered by the United States Department of Agriculture (USDA) (National AgrAbility Project, 2021). AgrAbility is currently funded in 24 states and has assisted over 10,000 farmers in the U.S. affected by physical, sensory, cognitive, and emotional disabilities (Fetsch et al., 2020a). Previous evidence has demonstrated that with the support of AgrAbility, farmers with disabilities have been able to complete up to $86 \%$ of farm responsibilities more successfully (Hamm et al., 2012).

Many farmers with disabilities receive assistance from individuals who can help them with physical tasks, also known as caregivers. A family member is the most common caregiver (Jepsen et al., 2011). However, friends, distant relatives, and other farmworkers have also been reported to serve in this role (Fetsch et al., 2020b). Despite this, little is known about the resources and support needed to ensure that caregivers can successfully navigate these responsibilities. On this point, Christen and Fetch (2008) suggested that communication is key to empowering the caregiver network. However, little is known about the techniques, strategies, and processes that lead to caregiver empowerment in North Carolina.

\section{Theoretical and Conceptual Framework}

Zimmerman's (1995) empowerment theory served as the theoretical framework guiding this investigation. Since 2011, the North Carolina AgrAbility Project, administered by the North Carolina A\&T State University Cooperative Extension Service, has provided resources and support to empower caregivers of farmers with a disability. Empowerment has been defined as a process by which individuals gain mastery over issues that concern them (Rappaport, 1987). Zimmerman (1995) theorized that empowerment involves three interconnected tenets (a) intrapersonal; (g) interactional; and (c) behavioral .

Intrapersonal empowerment relates to an individual's thoughts about themselves and their ability to influence others. As such, it affects an individual's affective dimensions such as their perceived control, self-efficacy, motivation, and mastery over issues and problems 
(Zimmerman, 1990, 1995). Interactional empowerment refers to an individual's understanding of their environment and how to navigate this context to achieve a desired outcome successfully. Through this critical awareness, they are then able to "exert control in their environment" (Zimmerman et al., 1990, p. 589) and mobilize relevant resources. The final tenet, behavioral, reflects the direct actions that individuals take to affect outcomes. It also includes behaviors that help them manage stress and adapt to change (Zimmerman, 1995). Figure 1 provides a visual model of the tenets of Zimmerman's empowerment theory.

\section{Figure 1}

Adapted from Zimmerman's (1995) Empowerment Theory

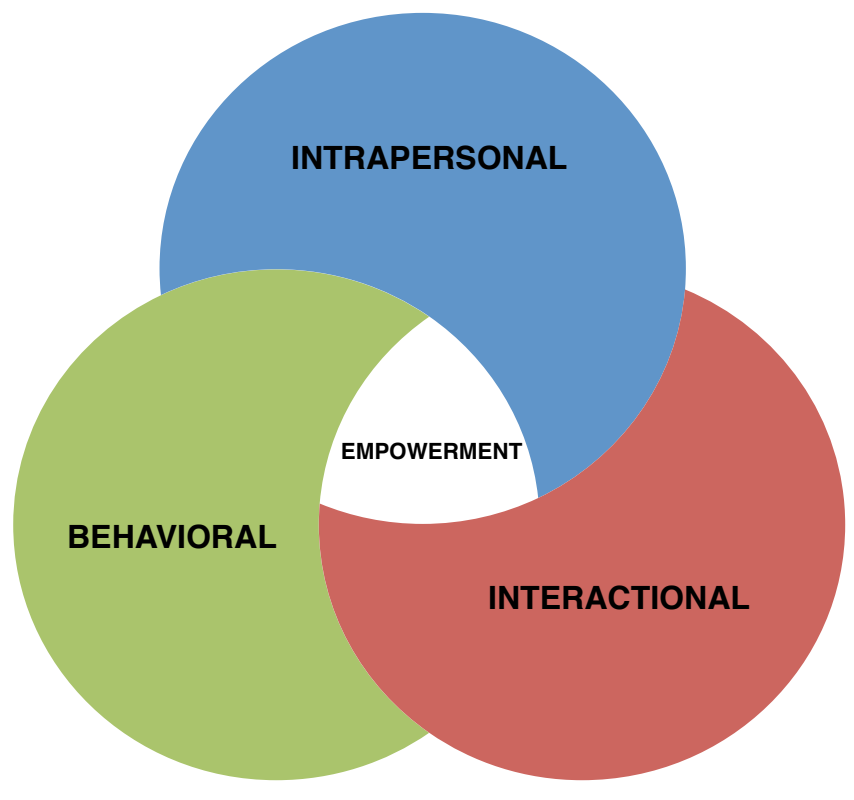

Using this lens, we investigated how the caregivers of farmers with a disability were empowered as a result of participation in the North Carolina AgrAbility Project by analyzing their self-reported experiences and those observed during data collection. For instance, we sought to understand caregivers' changes regarding self-efficacy, motivation, and perceived competence. We also probed how they learned to navigate their context as well as other personal and professional factors. As a result of the analysis, Zimmerman's (1995) empowerment theory served as a useful lens for investigating the empowerment of caregivers.

\section{Purpose}

The purpose of this study was to examine the ways in which caregivers of farmers with a disability have been empowered through the North Carolina AgrAbility project. 


\section{Methods}

The current study was nested, methodologically, in Stake's (1995) instrumental case study approach. Case studies allow researchers to describe a phenomenon in a bounded context using a variety of data sources. In particular, we bounded the case by place and time. To accomplish this, we used a criterion-based sampling procedure by which the North Carolina AgrAbility Project Director nominated individuals to participate who: (a) served as a caregiver to a farmer with a disability; and (b) had participated in the North Carolina AgrAbility Project in the past three years. Using this approach, three individuals agreed to participate.

Participant \#1 was a 67-year-old wife of a veteran farmer who was a Vietnam War veteran with a right arm amputation, diabetes, and other health limitations. The two main commodities produced on their farm were beef cattle and grass hay. Participant \#2 was a 57-year-old father of a farmer who had a spinal cord injury that resulted in him being in a quadriplegic state. The main commodities produced on their farm were sunflowers, soybeans, and poultry. Finally, Participant \#3 was a 31-year-old, long-term girlfriend of a farmer who had a rare neurological condition that began in 1988, which resulted in lower-body paralysis that affected his ability to walk. They mainly focused on large-scale vegetable production.

\section{Reflexivity}

When conducting qualitative research, the biases and experiences of researchers often influence the investigation (Houghton et al., 2013). Therefore, it is essential to reveal that the lead researcher worked with the North Carolina AgrAbility Project as a graduate research assistant. In this role, he assumed several responsibilities, such as recruiting new participants, educating the public on the mission of AgrAbility, and collecting data regarding farm injuries and deaths in North Carolina. The other researchers in this investigation were faculty at North Carolina A\&T State University at the time of data collection. Further, two of the researchers served as the lead investigator and Director for the North Carolina AgrAbility Project. Because the director of the program nominated individuals for participation, this could have influenced the responses received during our collection of data. As a result, these experiences and biases allowed us to obtain unique access to the phenomenon under investigation, which likely influenced our interpretation of the data.

\section{Data Sources, Analysis, and Rigor}

In the qualitative paradigm, ensuring rigor is critical. To achieve such, Lincoln and Guba (1985) advanced four standards to enhance rigor: credibility, transferability, dependability, and confirmability. We achieved these standards through (a) prolonged and varied field experiences in which we conducted on-site observations with each participant for three days; (b) maintaining thorough fieldnotes and observations; (c) employing member checks; and (d) a thick description of our findings. In addition to participant interviews, we also collected multiple sources of data to triangulate findings, including observations, and relevant program artifacts such as AgrAbility curriculum, pamphlets, and written correspondence. 
After the data collection, we used Corbin's and Strauss' (2015) constant comparative method to analyze each source of data. In the first stage of analysis, we employed open coding. During open coding, we become intimate with the data by analyzing sources line-by-line. We also coded data from various frames of reference and created memos based on conceptual and theoretical assertions (Corbin \& Strauss, 2015). As a result, the open coding process allowed us to discover the meaning hidden in data (Walker \& Myrick, 2006).

For the second phase of analysis, we employed axial coding (Corbin \& Strauss, 2015). In this stage, we reconstructed the data based on similarities by grouping our open codes into categories and subcategories. We then reduced the data using Walker's and Myrick's (2006) three recommendations by which we considered (a) the conditions in which the data were collected; (b) the actions and interactions of participants in response to the phenomenon; and (c) the consequences of actions taken by participants. This analytic technique helped us distill the data corpus into distinct categories as we began to weave our emergent findings into a coherent storyline (Corbin \& Strauss, 2015). In the final phase of analysis, we used to selective coding to transform the categories into substantive, theoretically rich explanations of the data. Using this analytic process, our findings emerged through four themes.

\section{Findings}

In our analysis of the data, we gained deeper insight into the phenomenon. Our findings demonstrated the caregivers' empowerment was largely shaped by how they reckoned with key barriers. Consequently, our emergent findings are presented through four themes of meaning (a) barriers to empowerment; (b) intrapersonal empowerment; (c) interactional empowerment; and (d) behavioral empowerment.

\section{Theme \#1: Barriers to Empowerment}

Through our observations of caregivers, we noted they often experienced barriers that hindered them from being empowered while receiving assistance from the North Carolina AgrAbility Project. In particular, the barriers to empowerment included (a) lack of trust; (b) poor communication skills; and (c) insufficient training. For example, when receiving assistance from the North Carolina AgrAbility Project, noticing positive results required time for the caregivers. While waiting for resources and equipment, some participants reported they initially experienced impatience and a lack of trust with AgrAbility. On this point, Participant \#2 explained that during his involvement with the program, he lost trust because as he was unable to observe changes for his son happening as quickly as he expected. Having poor communication skills was also a barrier to the empowerment of caregivers. Participant \#3 explained: "Some days are tuff, especially if we are bumping heads [with AgrAbility staff]. There is a need for better communication because we move at different paces."

The final barrier to the empowerment reported by the caregivers was a lack of perceived training among the AgrAbility staff. For instance, Participant \#1 described how she was frustrated with the program staff because at times because she perceived "workers lacked 
advanced training on how to work with farmers with a disability in the agricultural industry." This lack of trust appeared to be rooted in a disagreement between the caregivers and AgrAbility staff regarding the appropriate accommodations for farmers with a disability.

\section{Theme \#2: Intrapersonal Empowerment}

Although the caregivers experienced the aforementioned barriers, over time they learned to navigate such to become empowered through the North Carolina AgrAbility Project. The caregivers accomplished this through the use of internal processes by which they influenced themselves and others, i.e., intrapersonal empowerment (Zimmerman, 1995). As such, the caregivers experienced intrapersonal empowerment through (a) perceived control; (b) selfefficacy; (c) motivation; and (d) growth in competence.

Perceived control represented how the caregivers gained control over their actions, behaviors, and mindsets to better support farmers with a disability. As an illustration, Participant \#3 explained that at times, "caregiving can be exhausting both physically and mentally." However, through supports provided by the North Carolina AgriAbility Project, Participant \#3 stated, "I learned to control [myself] in various situations and better support [my boyfriend]." During observations, Participants \#1 and \#2 made significant strides in learning to control their environments when faced with challenges. For example, after receiving resources and support from the North Carolina AgrAbility Project, we noted that Participant \#1 and \#2 used various assistive technologies and strategies obtained through the program, which helped them support their farmer with a disability more efficiently.

The caregivers also reported making significant strides in improving their self-efficacy. For example, before AgrAbility's assistance, Participant \#2 reported "little to moderate" levels of self-efficacy to assist farmers with disabilities with duties on the farm. During observations, we also noted that Participant \#1 was not confident in her ability to make accommodations for her husband. However, after receiving assistance from the North Carolina AgrAbility Project, many caregivers reported greater levels of self-efficacy. To this point, Participant \#1 explained, "a Bump N' Drive gate that was provided by North Carolina AgrAbility was more for me than him because it allowed him to become more independent since he no longer had to rely on me for opening gates on the farm." We also observed Participant \#2 exhibiting more confidence after his son received a mechanical lift from North Carolina AgrAbility to help board his tractor, which reduced the stress of lifting his son for the aging caregiver.

Improved motivation also helped caregivers became empowered interpersonally, which reflected whether they had a desire to improve their ability to provide assistance as a caregiver for farmers with a disability. It should be noted that each participant in this study reported that they gained intrinsic motivation as a result of their participation in the North Carolina AgrAbility Project. For instance, Participant \#1 and \#2 maintained that AgrAbility made significant improvements in helping their farmers "gain independence" to complete on-farm tasks. As a result of these improvements, the caregivers' motivation improved because they desired to make additional progress. Case in point, after her boyfriend received an all-terrain vehicle (ATV) that greatly improved his mobility, Participant \#3 argued she become more interested in 
additional resources and educational opportunities through AgrAbility so that she could provide the best support possible. To this point, each of the caregivers in this investigation articulated that attending professional development opportunities coordinated by the North Carolina AgrAbility Project kept them engaged and motivated to advocate for disabled farmers. Participant \#1 explained, "I am always excited to go to events [through AgrAbility] and see things that allow it to be easier for people with a disability."

Finally, the caregivers also reported a growth in competence. After receiving assistance from AgrAbility, the caregivers explained they felt more competent in using assistive technology and research-based strategies to assist their farmers more effectively. For example, through incorporating ATV's, Bump N' Drive Gates, mechanical lifts, and other assistive technology, the physical demands placed on caregivers were often reduced, which helped them give more attention to other areas of need.

All three caregivers also said they felt "more educated" about best practices regarding farm safety, which helped them to provide guidance to other farmworkers. Participant \#1 explained, "[I now know] about farm safety and how it is practiced. I have male children who assist my husband as well. So, this has really helped." In the second theme, therefore, we demonstrated how the caregiver experienced intrapersonal empowerment through four sources: perceived control, self-efficacy, motivation, and growth in competence, after receiving assistance from the North Carolina AgrAbility Project.

\section{Theme \#3: Interactional Empowerment}

The third theme, interactional empowerment, reflected how individuals began to understand how to navigate their context to address issues and problems as a result of AgrAbility. Participants reported that interactional empowerment was experienced by developing and transferring skills and mobilizing resources. For instance, the caregivers began to develop and transfer the skills needed to accommodate farmers with disabilities better while also becoming knowledgeable about ways to manage resources effectively. Before receiving assistance from the North Carolina AgrAbility Project, all participants reported low skill attainment. However, after attending professional development sessions, Participant \#2 explained that he acquired the skills needed to "lift his son onto farm equipment safely." He also demonstrated how he learned to use a wrench and cable wires to lift his son onto a tractor through education provided by AgrAbility during our observations.

The caregivers also became empowered by learning to mobilize available resources. During the interview with Participant \#3, for example, she pointed to an old ladder that she previously used to help her boyfriend mount tractors and other farm machinery. She stated, "I would have to go up the ladder with him, lift his legs, step by step until he was high enough to get in the combine." After receiving assistance from AgrAbility, however, the caregivers demonstrated greater resource mobilization. In particular, Participant \#1 and \#3 shared they felt more empowered after learning to use assistive technologies such as an ATV and a mechanical lift as well as a new organizational structure for their barn recommended by AgrAbility professionals to ensure that their farm activities could operate more smoothly. As a result of learning to 
mobilize these tangible and intangible resources, Participant \#3's boyfriend gained most of his independence back, allowing her to focus on other tasks. Our observations also triangulated the interactional empowerment of caregivers. As such, we witnessed each caregiver in the investigation develop and transfer skills as well as mobilize resources to achieve goals.

\section{Theme \#4: Behavioral Empowerment}

The final theme, behavioral empowerment, reflected the caregivers' specific actions to positively impact the professional lives of farmers with a disability. The caregivers experienced this form of empowerment through organizational participation and community engagement. Organizational participation referred to how the caregivers became actively engaged in the disability community because of AgrAbilty. Participant \#3 explained, "I am the only one with a smartphone, so I keep in contact [with others in the AgrAbility]...to stay on top of certain things and events." Participant \#1 also expressed that she became the "primary source of communication" with the disability community through more engagement with the AgrAbility network. Further, Participant \#1 and \#3 also regularly attended disability-focused events out of their geographical region, such as the National AgrAbility Training Workshop, which helped them learn new strategies for their farm and share their experiences with others.

The caregivers also reported they became more engaged in their local and professional communities as a result of the North Carolina AgrAbility Project. For instance, Participant \#2 explained that after observing the distinct benefits of AgrAbility, they began to seek out more authentic experiences to connect with others in organizations such as the North Carolina Assistive Technology Program, Vocational Rehabilitation, and North Carolina Agromedicine Institute. She explained, "I've started accompanying my boyfriend when he attends farming conferences and receives certifications now." Meanwhile, Caregiver \#3 reported that she has now developed a "love for building relationships with other local farmers in the area" through attending community-based events. As a result of these connections, Participant \#1 illuminated that she felt more empowered because she developed the vocabulary to "better communicate" about her husband's needs.

\section{Conclusions, Discussion, and Recommendations}

The purpose of the study was to examine how caregivers of farmers with a disability had been empowered through the North Carolina AgrAbility project. When viewed through the lens of Zimmerman's (1995) empowerment theory, we conclude that although the caregivers reported key barriers, they were empowered at three distinct levels - intrapersonal, interactional, and behavioral. As a consequence, several implications and recommendations emerged.

First, because the participants reported a lack of trust, poor communication skills, and insufficient training of AgrAbility staff, we concluded that such served as barriers to the caregivers' empowerment. This finding is supported by the findings of Fetsch et al. (2020a) and Fetsch and Turk (2018). As such, we recommend that North Carolina AgrAbility provide tailored training and professional development for staff regarding how to best communicate with 
caregivers as well as farmers with a disability. We also recommend that North Carolina AgrAbility embed listening sessions into professional development opportunities so that caregivers can be given a space to articulate their concerns and staff can respond accordingly (Roberts et al., 2020a). Finally, additional research should seek to understand how caregivers could better navigate barriers to become empowered through the North Carolina AgrAbility Project and better sustain the agricultural pipeline for future generations (Alston et al., 2019, 2020). Future studies should also examine caregivers' preferences regarding receiving information about accommodating disabled farmers.

Despite the aforementioned barriers, we conclude the caregivers learned to overcome these challenges to become empowered on the intrapersonal level (Zimmerman, 1995). For example, the caregivers reported growth regarding their perceived control, self-efficacy, motivation, and competence. Such a notion does not appear to have been reported in the broader literature. As a result, these outcomes generated several potential questions that could be addressed through additional research: In what ways can caregivers' intrapersonal empowerment be leveraged to better diffuse AgrAbility in other states? Is caregivers' sense of intrapersonal empowerment stable over time? and Does the intrapersonal empowerment of caregivers extend to the farmers with a disability whom they provide assistance?

In the third theme, interactional empowerment, the caregivers learned to navigate their context to address complex issues and problems. We concluded the caregivers were able to accomplish this by developing and transferring skills as well mobilizing resources - a notion that has not been reported in previous literature on AgrAbility. Because of the importance of skill development, we recommend that additional educational and professional development opportunities be created that target the needs of the caregiver network based on their career phase (Roberts et al., 2020b). Through additional engagement opportunities at conferences, exhibits, and agricultural shows, the caregivers could gain access to unique resources and innovations in assistive technology. Consequently, we recommend that additional work be conducted to understand the types of marketing and communication approaches that would likely motivate caregivers of farmers with a disability to engage in outreach programs coordinated by the North Carolina AgrAbility Project.

Finally, we conclude the caregivers became behaviorally empowered through greater organizational participation and community engagement. As articulated by participants in this study, caregiving can be physically and mentally exhausting, which results in unique burdens placed on this population. Therefore, we recommend that AgrAbility create a formal support group for caregivers of farmers with a disability. Through support groups, caregivers could congregate and share practical solutions to navigate the physical, emotional, and cognitive demands of this role. Further, because learning to communicate about farmers with disabilities needs was critical to the empowerment of caregivers in this study, participation in support groups might help these individuals learn to express themselves to individuals who understand their experiences on a personal level. Going forward, more research is also needed to understand how innovations that could positively impact the lives of farmers with a disability might diffuse more rapidly (Rogers, 2003) if buy-in is achieved from the caregiver population. 


\section{Acknowledgements}

This research was partially funded by the USDA-NIFA National AgrAbility Project.

\section{References}

Alston, A. J., Roberts, R., \& Warren English, C. (2019). Building a sustainable agricultural career pipeline: Effective recruitment and retention strategies used by colleges of agriculture in the United States. Journal of Research in Technical Careers, 3(2), 1-23. https://doi.org/10.9741/2578-2118.1073

Alston, A. J., Roberts, R., \& Warren English, C. (2020). Toward a holistic agricultural student recruitment model: A national analysis of the factors affecting students' decision to pursue an agricultural related degree. Journal of Research in Technical Careers, 4(1), 128. https://digitalscholarship.unlv.edu/irtc/vol4/iss1/1/

Christen C. T., \& Fetsch, R. J. (2008). Colorado AgrAbility: Enhancing the effectiveness of outreach efforts targeting farmers and ranchers with disabilities. Journal of Applied Communications, 92(1), 57-73. https://doi.org/10.4148/1051-0834.1216

Corbin, J., \& Strauss, A. (2015). Basics of qualitative research: Techniques and procedures for developing grounded theory (4th ed.). Sage.

Fetsch, R. J., Leathers, C. L., \& Morgan, G. A. (2020a). Why do some farmers and ranchers overcome limitations from accidents and illnesses and others do not? AgrAbility works for most. Medical Research Archives, 8(2), 1-5. https://doi.org/10.18103/mra.v8i2.2047

Fetsch, R. J., Petrea, R. E., Jones, P. J., Field, W. E., \& Aherin, R. A. (2020b). A 25-year overview of AgrAbility demographics. Journal of Agromedicine, 25(3), 1-11. https://doi.org/10.1080/1059924X.2020.1837318

Fetsch, R. J., \& Turk, P. (2018). A quantitative assessment of the effectiveness of USDA's AgrAbility project. Disability and Health Journal, 11(2), 249-255. https://doi.org/10.1016/j.dhjo.2017.10.004

Hamm, K. E., Field, W. E., Jones, P. J., Wolfe, A., \& Olson, E. (2012). Twenty years of AgrAbility: A retrospective forum. Journal of Agromedicine, 17(4), 410-414. https://doi.org/10.1080/1059924X.2012.713844

Houghton, C., Casey, D., Shaw, D., \& Murphy, K. (2013). Rigor in qualitative case-study research. Nurse Researcher, 20(4), 12-17. https://journals.rcni.com/nurse-researcher/rigour-inqualitative-casestudy-research-nr2013.03.20.4.12.e326 
Jepsen, S. D., McGuire, K., \& Poland, D. (2011). Primary caregiver for a farm family member. The Ohio State University Extension. https://citeseerx.ist.psu.edu/viewdoc/download?doi=10.1.1.225.2551\&rep=rep1\&type=p $\underline{\mathrm{df}}$

Lincoln, Y., \& Guba, E. (1985). Naturalistic inquiry. Sage.

National AgrAbility Project. (2021). The vision of AgrAbility. Purdue University. http://www.agrability.org/

Rappaport, J. (1987). Terms of empowerment/exemplars of prevention: Toward a theory for community psychology. American Journal of Community Psychology, 15(2), 121-148. https://doi.org/10.1007/BF00919275

Roberts, R., Stair, K. S., \& Granberry, T. (2020a). Images from the trenches: A visual narrative of the concerns of preservice agricultural education teachers. Journal of Agricultural Education, 61(2), 324-338. https://doi.org/10.5032/jae.2020.02324

Roberts, R., Witte, B. M., Stair, K. S., Blackburn, J. J., \& Smith, H. E. (2020). The dimensions of professional development needs for secondary agricultural education teachers across career stages: A multiple case study comparison. Journal of Agricultural Education, 61(3), 128-143. https://doi.org/10.5032/jae.2020.03128

Rogers, E. M. (2003). Diffusion of innovations (5th ed.). Free Press.

Stake, R. (1995). The art of case study research. Sage.

Walker, D., \& Myrick, F. (2006). Grounded theory: An exploration of process and procedure. Qualitative Health Research, 16(4), 547-559. https://doi.org/10.1177/1049732305285972

Zimmerman, M. A. (1990). Taking aim on empowerment research: On the distinction between individual and psychological conceptions. American Journal of Community Psychology, 18(1), 169-177. https://doi.org/10.1007/BF00922695

Zimmerman, M. A. (1995). Psychological empowerment: Issues and illustrations. American Journal of Community Psychology, 23(5), 581-599. https://doi.org/10.1007/BF02506983

(C) 2021 by authors. This article is an open access article distributed under the terms and conditions of the Creative Commons Attribution license (http://creativecommons.org/licenses/by/4.0/). 